\title{
DER WORTSCHATZ ALS "WAHRHEITSVERMITTLER" IM AUBENPOLITISCHEN PRESSEKOMMENTAR
}

\section{Einleitung}

Bekannterweise weist die ausgewählte journalistische Textsorte eine spezifische textuelle Struktur auf; ihre inhaltliche (evaluative) und intentionale Komponente sind zunächst in Kürze zu erläutern. Es zeigt sich, daß die Bewertung als obligatorisches Element eines Kommentartextes textstrukturell und stilistisch-pragmatisch bedingt ist und daß sie im Prinzip mittels Lexik realisiert wird. Das Funktionieren der Lexik als Bewertungsträger wird anschließend aufgrund einer exemplarischen Fallanalyse dargestellt, wobei hauptsächlich Zusammenhänge zwischen textstrukturellen Besonderheiten der Kommentartexte und stilistisch-pragmatischen Eigenschaften der Lexik interessieren.

\section{Zum Wesen des Kommentierens und des Kommentars}

Den kommunikationswissenschaftlichen ${ }^{1}$ und kommunikationspsychologischen ${ }^{2}$ Erkenntnissen entsprechend, wohl aber auch in Übereinstimmung mit dem allgemein verbreiteten und konventionell festgelegten Verständnis der Sprechtätigkeit des Kommentierens $^{3}$ sind Kommentare typische Vertreter derjenigen journalistischen Darstellungsformen, welche persönliche, subjektive, bewertende Stellungnahmen, Meinungen und Einstellungen zum thematisierten Sachverhalt in die Öffentlichkeit zu lancieren haben. Die Sprechtätigkeit des Kommentierens gehört neben Informieren somit $\mathrm{zu}$ den grundlegenden und unabdingbaren Bestandteilen der massenmedialen Berichterstattung. Allerdings lassen sich das Informieren und das Kommentieren nicht

1 Vgl. z. B. Noelle-Neumann/Schulz/Wilke (1994).

2 Gemeint ist das Kommentieren als Verhaltensmuster im komplexen Kommunikationsverhalten des Menschen. Man erkennt es daran, daß der Adressat "auf die Rezeption einer Information mit einer Stellungnahme" reagiert (Posner 1980, 2).

3 Kommentiert wird regelmäßig auch in alltäglicher privater Kommunikation, und hier gilt, daß dabei persönliche, subjektive und insbesondere bewertende Anmerkungen gemacht bzw. Stellungnahmen zum kommentierten Sachverhalt abgegeben werden. 
als isolierte Komponenten sondern zwangsläufig als miteinander eng verbundene und wechselseitig abhängige Aufgaben der öffentlichen Kommunikation interpretieren. Kommentiert wird notwendigerweise vor dem Hintergrund des Informierens, was zur Folge hat, daß die textuelle Struktur eines Kommentartextes in der Regel eine informative Orientierung über kommentierte Sachverhalte beinhaltet. In gleicher Weise will auch das mediale Informieren auf kommentierende Inhalte üblicherweise nicht verzichten. Darüber hinaus kann die Sprechtätigkeit des Kommentierens auch als Informieren über die Meinung verstanden werden; es ist folglich das Informieren über des Autors Meinungen und Einstellungen zu den thematisierten Ereignissen und/oder Sachverhalten. Allerdings ändert sich dabei die Qualität des Informierens, der Charakter der Information im Sinne der modifizierten Betrachtungsperspektive und der damit verbundenen Absichten des Autors bei gleichbleibendem Kommunikationsgegenstand. Im Idealfall wechselt zugleich die Präsentationsform, so daß die Informationen über Einstellungen in den sogenannten interpretativen bzw. meinungsbetonten und meinungsbildenden journalistischen Textsorten erscheinen, welche sehr oft eben der journalistische Kommentar repräsentiert. ${ }^{4}$

Werden Texte aus der Sicht der Textlinguistik als Realisierungen bestimmter Textmuster aufgefaßt, so befolgt auch der journalistische Kommentar ein konventionell festgelegtes und prototypisches Muster. ${ }^{5}$ Wie schon oben ersichtlich, beinhaltet dieses hauptsächlich den Ausdruck der Meinung. Da jedoch ein jeder Ausdruck der Meinung unbedingt auch evaluativ ist, zählt das Bewerten zu den obligatorischen Komponenten der kommentarspezifischen textuellen Struktur. In handlungstheoretischer Hinsicht nimmt es innerhalb der Textstruktur eine zentrale Position ein, es ist die vorherrschende Sprachhandlung, welche als eine Art Superhandlung alle anderen Handlungen im Kommentartext dominiert (Lenk 1986, Lüger 1995). Es kommt als eigenständiges Element im Sinne von Bewertungshandlung oder Bewertungssequenz vor, es kann sich

4 In der Tat dienen nicht nur Kommentare und kommentarähnliche meinungsbildende journalistische Textsorten (Leitartikel, Glosse, Kolumne, Kritik) der medialen Meinungsäußerung und Meinungsbildung. Auch primär informative journalistische Textsorten (Nachricht, Meldung, Bericht) kommen üblicherweise ohne die bewertenden und beurteilenden Elemente bzw. Passagen nicht aus, zumal man bereits die Selektion von Informationen in den Nachrichten als meinungsbildend bzw. meinungsbeeinflussend interpretieren kann. Interessantes hierzu bringt Schneider $(1984,281$ ), wonach Kommentare und andere meinungsbetonte Textsorten überhaupt nicht diejenigen medialen Texte sind, in denen in erster Linie die Meinungsäußerung und -beeinflussung vollzogen werden. Im Gegenteil: Meinungen werden "in ungleich höherem Grade durch die Auswahl und Aufbereitung von Informationen gesteuert". Der Selektion von Informationen ist folglich $u$. a. eine Steureungsfunktion in der Meinungsbildung zuzuschreiben.

5 Es kann angenommen werden, daß die inhaltlich-textuelle Musterhaftigkeit eines Kommentartextes auch von der Rezipientenseite weitgehend erkannt wird. Dies ergibt sich bereits aus dem allgemeinen Verständnis der Sprechtätigkeit des Kommentierens, insbesondere aber aus den Erfahrungen mit der medialen Berichterstattung, über die ein regelmäßiger Zeitungsleser in der Regel verfügt. Seinerseits gelten somit verbalisierte Meinungen und Stellungnahmen zu den aktuellen und öffentlich relevanten Sachverhalten als erwartete Qualitäten eines journalistischen Kommentars. 
aber auch um ein Prinzip handeln, welches die gesamte Kommentartextstruktur durchdringt (Läzer 1988, Ramge 1994). In Kommentartexten werden somit komplexe Bewertungshandlungen realisiert (Läzer 1988), und zwar intentional mit dem Hauptziel, daß der Rezipient sie akzeptiert und übernimmt (Schneider 1984, Läzer 1994, Lüger 1995). Der persuasive Charakter läßt sich auf die kommentarspezifische Textintention zurückführen, die, wie schon oben angedeutet, hauptsächlich im Appellieren besteht (Lüger 1995). Die intendierte Reaktion des Rezipienten ist die Übernahme der im Kommentar ausgedrückten bewertenden Positionen. ${ }^{6}$ Aus der Perspektive des Kommentators bestehen die kommentarspezifischen persuasiven Aspekte darin, "Strategien zu verfolgen, die geäußerten Bewertungen als begründet, gerechtfertigt, folgerichtig, kurz: als einsichtig erscheinen zu lassen" (Läzer 1994, 124). Begründungen, Rechtfertigungen, Erläuterungen u. ä. existieren somit als kommentarspezifische subsidiäre Handlungen, um die Akzeptierensbedingungen beim Rezipienten zu verbessern, um die Gültigkeit der zentralen bewertenden Aussage zu untermauern, unterstützen, argumentieren und so für den Rezipienten verständlicher, akzeptabler und ausführbarer zu machen. Sie sind Elemente der kommentarspezifischen argumentativen Textstruktur.

Die persuasiven Strategien der Textgestaltung bedienen sich hauptsächlich sprachlicher Einheiten und sprachlicher Strukturen, um dadurch die intendierten Effekte beim Rezipienten zu erreichen. ${ }^{7}$ Das Evaluativ-Persuasive im Kommentartext läßt sich somit primär auf der lexikalischen Ebene beobachten: Entweder ist es an expliziten sprachlichen Indikatoren erkennbar (deutlich bewertende Lexik, Vergleiche o. ä.) oder es beruht auf der pragmatischen Komponente der Sprachverwendung. Durch die Wahl der Lexik ist es möglich, bestimmte Einstellungen und Wertungen beim Rezipienten verdeckt zu suggerieren, und dieser erkennt sie in der Regel erst durch bewußte Umdeutungen bzw. Uminterpretationen.

Die lexikalische Ausprägung der Kommentartexte ist jedoch nicht nur auf die besprochene kommentarspezifische textuelle Struktur zu beziehen; ebenso betrifft sie allgemein gültige Gesetzmäßigkeiten der sprachlichen Kommunikation. Demzufolge ist sie von (mindestens) zwei wesentlichen Standpunkten her zu beurteilen: 1. vor dem Hintergrund der konventionellen Kommentartextstruktur, und 2. unter Berücksichtigung der für die Kommunikation schlechthin geltenden Prinzipien einer wirksamen sprachlichen Gestaltung von Texten. Der Kommentator gestaltet seinen

6 Die Auffassung des Kommentars als Textsorte mit persuasiven Zielen ist zwar stark medial und situativ bedingt, sie nähert sich aber zugleich denjenigen Auffassungen der sprachlichen Kommunikation, die das Kommunizieren schlechthin primär im Sinne der Rezipientenbeeinflussung verstehen und interpretieren (vgl. Keller 1990, von Polenz 1991).

7 Von der intendiert persuasiven Einsetzung des Bildes, die insbesondere in der Boulevard-Presse praktiziert wird, wird hier abgesehen, da auch der besprochene Beispieltext einem anderen Zeitungstyp, nämlich der überregionalen Tagespresse entstammt. Das bedeutet allerdings nicht, daß die sogenannte seriöse Presse auf sensationelle Aufmachung und intentionsgestützte Verwendung des Bildes verzichtet. 
Text nämlich nicht nur nach den Prinzipien des Kommentierens und des Kommentars als Textsorte, er realisiert nicht nur ein spezifisches Textmuster, sondern er schreibt auch einfach einen Text. Dabei bemuiht er sich aber um eine optimale, in der Regel um eine lesewerbende und attraktive sprachliche Gestaltung, er strebt nach einer stilistisch und pragmatisch angemessenen Ausdrucksweise. Es ist hierbei anzunehmen, daß die Formen von sprachlichen Bewertungen im Kommentar auch aus den Bemühungen um einen stilistisch guten Text resultieren und daß sie insofern mit der Sprechtätigkeit des Kommentierens nur indirekt $\mathrm{zu}$ tun haben (Ramge 1994). Die obligatorische Bewertungskomponente eines Kommentartextes läßt sich somit in doppelter Hinsicht interpretieren: Den verbalisierten oder interpretativ gewonnenen Bewertungen liegen entweder kommentarspezifische strukturinterne Prinzipien der Textmusterrealisierung zugrunde oder sie beruhen auf strukturexternen stilistisch-pragmatischen Prinzipien der Textgestaltung. Allerdings lassen sich beide Prinzipien wiederum nicht strikt voneinander trennen: Eher korrespondieren sie miteinander.

\section{Eine Fallanalyse}

Im folgenden wird versucht, die obigen Überlegungen $\mathrm{zu}$ den bewertenden und intentionalen Komponenten der Kommentartexte an einem Beispieltext zu überprüfen. Es wird dabei hauptsächlich der Frage nachgegangen, inwiefern sich nun Zusammenhänge zwischen den kommentarspezifischen Textsorteneigenschaften und der lexikalischen Ausprägung eines konkreten Kommentartextes identifizieren lassen. Erfolgt die Selektion der Lexik im Prozeß der Textgestaltung primär als strategiegeleitetes Handeln des Kommentators, wodurch er die textintentional begründete kommunikative Funktion der betreffenden Textsorte verfolgt oder ist die jeweilige Sprachwahl in erster Linie stilistisch-pragmatisch begründet und somit als Qualität des Individualstils interpretierbar? 


\title{
Seelenlos und am Boden zerstört
}

\author{
VON JENS SCHNEIDER
}

War es früher nicht schön hier? In Zagreb oder Ljubljana ist es heute nahezu verboten, diese Frage zu stellen. Auch in manchen Zirkeln in Sarajewo oder Belgrad gilt sie als Zeichen von Jugonostalgie oder gar Verrat. Es soll schon einige den Job gekostet haben, öffentlich an vielleicht gute alte'Zeiten zu erinnern. Also fragen die Menschen lieber leise: War es nicht beinahe schön damals, vor dem Krieg? Alle hatten ein Dach überm Kopf, oft ein sehr ansehnliches, und immer gab es zu essen - fett, würzig und reichlich. Das muslimische Bairam-Fest und zweimal Weihnachten gefeiert, jeden Sommer ans Meer - und Krieg? Krieg war in Afrika oder Beirut, aber nicht bei uns.

Derlei Nostaigie hat immer etwas Verlogenes, die Erinnerung verklärt den Blick. Vergessen wird der Mangel an Demokratie, das unterdrückte Nationalbewußtsein und der wirtschaftliche Verfall. Es wird verdrängt, daß die Fehler Titos eine Ursache des verheerenden Krieges waren. Es gibt kein Zurück, und das ist.wohl auch gut so. Dennoch drängt sich angesichts der Unterzeichnung des Dayton-Abkommens die Frage auf: Was haben die Jugoslawen für ihr fröhlichmarodes Regime eingetauscht?

Ode und verfallen, undemokratisch und moralisch verkommen, ethnisch gesäubert und dramatisch verarmt - das ist das neue Jugoslawien. Aufgesplittert in sechs Republiken; fünf hat der Verfall erfaßt, eine - Slowenien - ist davongekommen. Aber der Rest: Millionen Verlierer stehen einer Handvoll mafioser Kriegsgewinnler gegenüber, Hunderttausende starben, unzählige Menschen wurden vertrieben. Die Täter laufen frei herum, einige regieren.

Klinisch tot ist das multikulturelle Bosnien; Dayton hat diesen Zustand - trotz aller gutgemeinten Bekenntnisse zur Integrität des Landes - einstweilen besiegelt. Das bettelarme, zerstörte Land besteht de facto aus drei ethnisch weitgehend gesäuberten Gebieten, beherrscht von jeweils allmächtigen Ein-ParteienRegimen. Nur in wenigen Städten gibt es noch Spuren multikulturellen Zusammenlebens. Vorerst dürfte die Trennung der Volksgruppen sogar fortgesetzt werden, wenn Minderheiten lieber zu ihresgleichen ziehen. als unter feindseligen Regimen in der Heimat zu bleiben. Diese Aussicht dürfte auch viele Vertriebene von der Rückkehr abschrecken.

Nicht vergleichbar, aber dennoch trostlos sieht es in den anderen Republiken aus: Manche wollen die Serben als Sieger sehen, weil ein Teil Bosniens von Serben beherrscht wird. Welch ein Sieg! Der Krieg und die Sanktionen haben Serbien und Montenegro an den Rand des Ruins gebracht; es herrscht beklemmende Armut. Kriminelle 'ofofpinieren 'das' Ge-

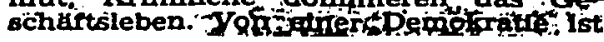

das Land weiter entfernt als vor dem Krieg. Ungelöst bleibt das Kosovo-Problem, wo die unterdrückten Albaner zunehmend unversöhnlich auf ihre Rechte pochen; daß diese Zeitbombe entgegen allen Vorhersagen nicht losgegangen ist, heißt nicht, daß sie entschärft wäre.

Fatal vernachlässigt schlingert die Teilrepublik Mazedonien in die Krise, gebeutelt von den Sanktionen, im Bestand bedroht durch Reibungen zwischen den Volksgruppen. Und wie lange wird der Jubel der Kroaten über ihren eigenen Staat noch anhalten? Tudjman mag sich als Sieger wähnen, hat er doch die Integrität des Landes hergestellt und ungestraft großen Einfluß in Bosnien gewonnen. Dafür hält er Kroatien im Würgegriff und regiert nach Gutdünken; Absahner si- 
chern sich in seinem Staat ihre Pfründe. Demokratie? Fehlanzeige. Armut quält auch die meisten Kroaten, immerhin läßt die Aussicht auf die Wiederbelebung des Tourismus hoffen.

Und dann ist da in allen Republiken noch ein Verlust, den niemand wahrhaben will: Mit der Vertreibung ganzer Volksgruppen haben auch die Vertreiber verloren, haben fast alle Regionen einen Teil ihrer Seele eingebüßt. Im ohnehin repressiven Klima herrscht kulturelle Odnis, dominiert schwulstiger Kitsch. Die Vielfalt mag die Geißel Jugoslawiens gewesen sein, sie war aber auch sein größter Reiz. Zu den Millionen Heimatlosen müssen auch jene gezählt werden, die sich bis heute als Jugoslawen verstehen und auf keiner Seite eine Heimat sehen. Das Ringen um Bewahrung ihrer Identität, wie es beispielhaft der geniale Regisseur Emir Kusturica versucht, scheitert, weil auf allen Seiten und auch außerhalb Jugoslawiens Bekenntnisse zu einer Seite verlangt werden.
Dayton hat dieses Lagerdenken erst einmal gefestigt. Die Väter des Krieges sitzen fester denn je im Sattel. Man hat sie für diesen Friedensschluß gebraucht und wird sie weiter brauchen, um den Waffenstillstand zu festigen. Bestenfalls birgt das Abkommen mit seinen Bekenntnissen zu Frieden und Demokratie das Potential in sich, die fürchterlichen Regime zu Übergangslösungen zu machen. Viele Jugoslawen sehnen sich danach: Bei aller Düsternis gibt es in Belgrad, Zagreb oder Sarajewo eine große Schar Kriegsmüder, die hoffen, daß der Westen bereit ist, nach dem militärischem auch ziviles Engagement zu zeigen. Gerade jetzt besteht eine geringe Chance: Die politischen Führer sind derzeit empfänglich für Druck. Den aber müBten Europa und Amerika leisten; und dazu braucht es einen langfristigen Einsatz von Personal und viel Geld für Wiederaufbauhilfe auf allen Seiten. Dieser hohe Preis des Friedens dürfte noch immer geringer sein als die Kosten -des nikchsten Krieges, , ,

Der Beispielkommentar wurde in der Süddeutschen Zeitung Ende 1995 veröffentlicht, und er thematisiert Verhältnisse auf dem Kriegsgebiet Balkan nach der Unterzeichung des Dayton-Abkommens. Der Titel Seelenlos und am Boden zerstört signalisiert bereits eindeutig evaluative Positionen des Kommentators, die im weiteren Text immer wieder zum Ausdruck kommen. Zugleich kündigt er das Textthema ${ }^{8}$ an: Es ist die Befürwortung des internationalen militärischen und zivilen Engagements, wođurch der geistige und materielle Verfall des ehemaligen jugoslawischen Gebietes gestoppt und eventuell überwunden werden kann. Die thematische Entfaltung ist allerdings komplexer. Sie reicht von den nostalgischen und satirisch-ironischen Erinnerungen an das ehemalige Jugoslawien, wobei das anscheinend Banale etwa vergangene aber gute alte Zeiten ins Bewußtsein zu rufen hat (immer gab es zu essen fett, würzig und reichlich) über die Problematisierung des ehemaligen fröhlichmaroden Regimes bis hin zur Verzweiflung am Friedensabkommen von Dayton. Zugleich fehlen aber auch ausführliche Darlegungen der Nachkriegsverhältnisse nicht. Makrostrukturell lassen sich im Text im wesentlichen drei thematische Einheiten erkennen: (1) Charakterisierung der politischen und wirtschaftlichen Verhältnisse im ehemaligen Jugoslawien, (2) eingehende Informationen zum Verfall einzelner Republiken und (3) Generalisierung dieses Zustands auf das gesamte Gebiet des 
ehemaligen jugoslawischen Staates. Erschließbar sind sie etwa aus folgenden Topikketten: (1) der Mangel an Demokratie - das unterdrückte Nationalbewußtsein der wirtschaftliche Verfall - die Fehler Titos - alles interpretierbar als Ursachen des verheerenden Krieges. Das bosnische Gebiet nach dem Krieg ist somit (2) öde und verfallen - undemokratisch - moralisch verkommen - ethnisch gesäubert - dramatisch verarmt - aufgesplittert - Imeist vom/ Verfall erfaßt - Millionen Verlierer Hunderttausende starben - unzählige Menschen wurden vertrieben - klinisch tot - das bettelarme zerstörte Land. Ähnliches gilt auch für andere ehemalige Republiken: Serbien und Montenegro befinden sich am Rand des Ruins - es herrscht beklemmende Armut - Kriminelle dominieren; Mazedonien ist fatal vernachlässigt - /es/ schlingert in die Krise - gebeutelt von den Sanktionen - im Bestand bedroht durch Reibungen zwischen den Volksgruppen. Auch Demokratie in Kroatien sei eine Fehlanzeige, denn Armut quält die meisten Kroaten. Im allgemeinen herrscht auf dem Gebiet des ehemaligen Jugoslawiens ein (3) repressives Klima - eine kulturelle Ödnis und es dominiert schwulstiger Kitsch.

Evaluatives ist in zitierten Belegen reichlich enthalten und entspricht wohl der spezifischen textuellen Struktur eines Kommentartextes. Ausführliche Erläuterungen samt expliziten Bewertungen lassen sich als Begründungen bzw. Argumentation der thematisierten Bemühungen um den Frieden in und vor allem nach Dayton interpretieren, was allerdings erst im letzten Absatz des Kommentars eindeutig zum Ausdruck kommt: Dayton hat dieses Lagerdenken erst einmal gefestigt - Die Väter des Krieges sitzen fester denn je im Sattel.- Bestenfalls birgt das Abkommen /../ das Potential in sich, die fürchterlichen Regime zu Übergangslösungen zu machen. Gerade jetzt besteht eine geringe Chance für ziviles Engagement...

Die zitierten Beispiele sind in erklärend-darlegenden Passagen des Textes plaziert. Es handelt sich eindeutig um Ausdrücke, die mit extrem negativen Konnotationen uiberlagert sind. Allerdings scheint es, daß sie nicht primär bzw. nicht nur in Funktion einer negativen Bewertung der thematisierten Sachverhalte selektioniert und eingesetzt worden sind. Zwar fungiert der zitierte Wortschatz im Prinzip als bewertende Benennung, stärker in den Vordergrund treten jedoch emotionale, expressive und assoziative Bedeutungskomponenten der Lexik. Die Aussagen werden somit nicht nur bewertet, sondern zusätzlich dramatisiert und emotionalisiert. Die Charakterisierung mit öde und verfallen, moralisch verkommen, ethnisch gesäubert, dramatisch verarmt, Millionen Verlierer, Hunderttausende starben, klinisch tot, das bettelarme zerstörte Land zielt nämlich eindeutig auf Emotionen. Es kann dabei jedoch die Annahme vertreten werden, daß diese mitkommuniziert werden eher als Unterstützung der bewertenden Haltungen und Stellungnahmen, die sozial reguliert sind und mittels deren auch persuasive Absichten verfolgt werden. Gemeint ist die Begründung bzw. Argumentation der thematischen Einheit, die im letzten Absatz des Beispieltextes verbalisiert wird: Die Verifizierung der Bemühungen um den Frieden auf dem Balkan, zumal am Textende sogar instruktive Aussagen hierzu getroffen werden. Weniger handelt es sich folglich nur um eine Mitteilung über individuelle emotional-subjektive 
Haltungen des Kommentators. Die Lexik fungiert somit nicht schlicht als Anzeichen des Individualstils des jeweiligen Autors; sie vermittelt auch die konventionell festgelegte und kulturell beeinflußte Wahrnehmung der "Wahrheit" über die räumlich, wohl aber auch sozial und psychologisch distanzierte Welt.

Aufgrund des Gesagten lassen sich mehrere Schlußfolgerungen ableiten: 1. die Lexik als Bewertungsträger wird im Text aus textstrukturellen und stilistischpragmatischen Gründen eingesetzt, 2. ihr Funktionieren im Text läßt sich u. a. handlungsbezogen bzw. textintentional und stilistisch-pragmatisch erklären. Allerdings zielt insbesondere die letztere Erklärung nicht unbedingt nur auf den Individualstil und auf die individuellen Absichten des Autors. Das stilistisch-pragmatische Potential der Lexik beinhaltet nämlich eher eine konventionalisierte und/oder angestrebte Sichtweise einer Sprachgemeinschaft als etwa persönliche und subjektive Einstellungen des jeweiligen Schreibers.

\section{Literatur}

HEUSINGER, S. 1995. Pragmalinguistik. Texterzeugung, Textanalyse; Stilgestaltung und Stilwirkungen in der sprachlichen Kommunikation. Ein Lehr- und Übungsbuch. Frankfurt am Main.

KELLER, R. 1990. Sprachwandel. Von der unsichtbaren Hand der Sprache. Tübingen.

LÄZER, R. 1988. Zur Illokutionsstruktur von Pressekommentaren - ein praktischer Beitrag zur Theoriendiskussion der handlungsorientierten Textanalyse. In: Zeitschrift für Germanistik 9, 472-479.

LÄZER, R. 1994. Persuasionsstragien im Wandel. Wertewandel und Textstrukturen in Kommentaren der DDR-Presse zur Zeit der 'Wende'. In: MOILANEN, M./L. TIITTULA (Hrsg.): Überredung in der Presse. Texte, Strategien, Analysen. Berlin, New York, 121-147.

LENK, H. 1986. Persuasionsstrategien in der Manipulation. Handlungsstrukturanalysen von Kommentaren der BRD-Presse. Diss. Berlin.

LÜGER, H.-H. 1995. Pressesprache. 2., neu bearbeitete Aufl. Tübingen.

NOELLE-NEUMANN, E./W. SCHULZ/J. WILKE (Hrsg.). 1994. Fischer Lexikon Publizistik Massenkommunikation. Frankfurt am Main.

von POLENZ, P. 1991. Deutsche Sprachgeschichte vom Spätmittelalter bis zur Gegenwart. Bd. 1. Berlin, New York.

POSNER, R. 1980. Theorie des Kommentierens. 2. Aufl. Wiesbaden.

RAMGE, H. 1994. Auf der Suche nach der Evaluation in Zeitungskommentaren. In: MOILANEN, M./L. TIITTULA (Hrsg.): Überredung in der Presse. Texte, Strategien, Analysen. Berlin, New York, 101-120.

SCHNEIDER, W. (Hrsg.) 1984. Unsere tägliche Desinformation. Wie die Massenmedien uns in die Irre führen. Hamburg. 
Kot je znano, izkazuje izbrana besedilna vrsta komentar značilno besedilno zgradbo. Le-ta temelji na vzorcu sporočanja, ki pomeni obširneje, s pojasnili, pripombami in ocenami poročati o čem. Vrednostne presoje so torej obvezne sestavine komentarja, ki ga komunikologija uvršča med besedilne vrste, opravljajoče vlogo oblikovanja javnega mnenja in prepričevanja naslovnika. $\mathrm{Z}$ vidika teorije govornih dejanj je komentar skupek hierarhično prepletenih govornih dejanj, v katerem prevladuje govorno dejanje vrednostne presoje predmeta sporočila. Sporočevalno oz. sporazumevalno namero komentarja je mogoče razumeti in razložiti tako, da govorec želi vplivati na naslovnika in povzročiti, da le-ta prevzame izrečene vrednostne presoje. $\mathrm{K}$ temu bistveno pripomore besedje, vendar vsakokratnega izbora besed ni smotrno razlagati le na osnovi značilne zgradbe in namena vsakokratnega besedila. Prav tako je odvisen od splošno veljavnih načel jezikovnega sporočanja, saj si govorec načeloma prizadeva za slogovno in pragmatično primerno oz. učinkovito ubeseditev predmeta sporočila. Obeh vidikov, ki narekujeta izbiro in rabo besed $v$ besedilu, ni možno popolnoma ločeno obravnavati, saj se prepletata. Na osnovi izbranega in analiziranega besedila pa je možno tudi sklepati, da besede, ki so že same po sebi nosilke vrednostnih sodb in vsekakor prispevajo $k$ tipični besedilni zgradbi in slogovno zaznamovani ekspresivnosti besedila, ne izražajo nujno osebnih sodb posameznega govorca, pač pa pogosto podružbljene, ustaljene oz. željene vrednostne presoje šiř̌ega jezikovnega okolja. Njihovo rabo v časopisnem komentarju je torej možno razlagati tudi na osnovi tovrstnih pragmatičnih zmožnosti. 